\title{
Research of the Principle of Standing Wave and Critical Speed Determination for the Radial Tire
}

\author{
Yunlong Wang ${ }^{1, a^{*}}$, Hongtian Zhang ${ }^{2, b}$ and Xiaojie Qi, \\ ${ }^{1}$ College of Power and Energy Engineering/Harbin Engineering University, Harbin, China \\ ${ }^{2}$ College of Power and Energy Engineering/Harbin Engineering University, Harbin, China \\ ${ }^{3}$ School of Automobile and Traffic Engineering/Heilongjiang Institute of Technology, Harbin, China \\ ayunlong14666@163.com, bzhanghongtian@126.com, c112930125@qq.com
}

Keywords: Radial Tire; Tire Standing Wave; Critical Speed; Finite Element Simulation

Abstract. To test standing wave of the tire, constructed the simulation model by ABAQUS software for radial tire running rapidly on the automotive chassis dynamometers, meanwhile assembled the automotive chassis dynamometers, high-speed camera and actual radial tire together. The experiment indicated that the critical speed of simulation tire is $255.83 \mathrm{~km} / \mathrm{h}$, actual tire $268.5 \mathrm{~km} / \mathrm{h}$, the difference is $4.95 \%$, Consistency is very good for simulation result and actual tire testing.

When the vehicle is running rapidly to the special speed, the tire will bring standing wave, while distortion of the tire largen, the temperature rise rapidly, material performance decline quickly, the tire will be destroyed at short notice. Now the method of critical speed research of the tire included analytical method, experimentation and finite element simulation, the analytical method is that constructed theory model of the tire ${ }^{[1]}$, for example Sakai Hideo researched critical speed of radial tire on the equation of girder vibration by loop girder model of elasticity radicle ${ }^{[2]}$. The experimentation method was that high speed photograph shot distortion picture of mini diameter tire model in the different speed by flywheel driving during standing wave ${ }^{[3]}$. The method of finite element simulation was that the tire model was constructed by software of finite element analysis, accordingly researched critical speed and its effect factor of the tire model ${ }^{[4-7]}$. These methods were advantages and disadvantages, the analytical method was more comprehensive but not enough precision. The experimentation method was enough precision but higher cost and more dangerous. The simulation method was more comprehensive but not enough precision.

\section{Radial tire modeled}

We research radial tire(Bridgestone 195/60 R15), constructed finite simulation model of tire by ABAQUS software, made the tire to run rapidly on the automotive chassis dynamometers. We constructed rotational symmetry model of the tire on ignoring flower pattern, defending line and sign line because of very complex structure of the tire, thus analysed the tire load and twirl. For the tire and wheel rim, the joint was predigested fixed restriction of relevant area of bead of tire.

Simulation parameter setup. We established interaction, selected main surface and secondary surface between each surface(selected rolling drum as main surface because we need set it as rigid body, main surface red, secondary surface pink). Then we continued to found attribute of touch action, selected tangential force from mechanics of attribute of touch action, friction formula as roughness. We established attribute of joint section radial pressure, eddy, else default(fig. 1). 


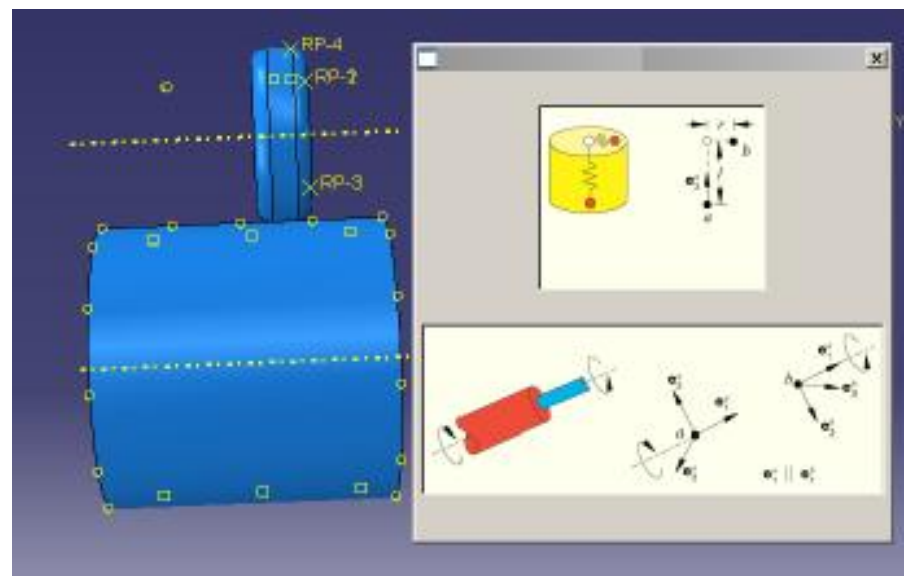

Fig. 1 found movement type

\section{Standing wave analysis of simulation model.}

Analysis task. We found task module and refered, entered result module, visualization result was fig. 2.
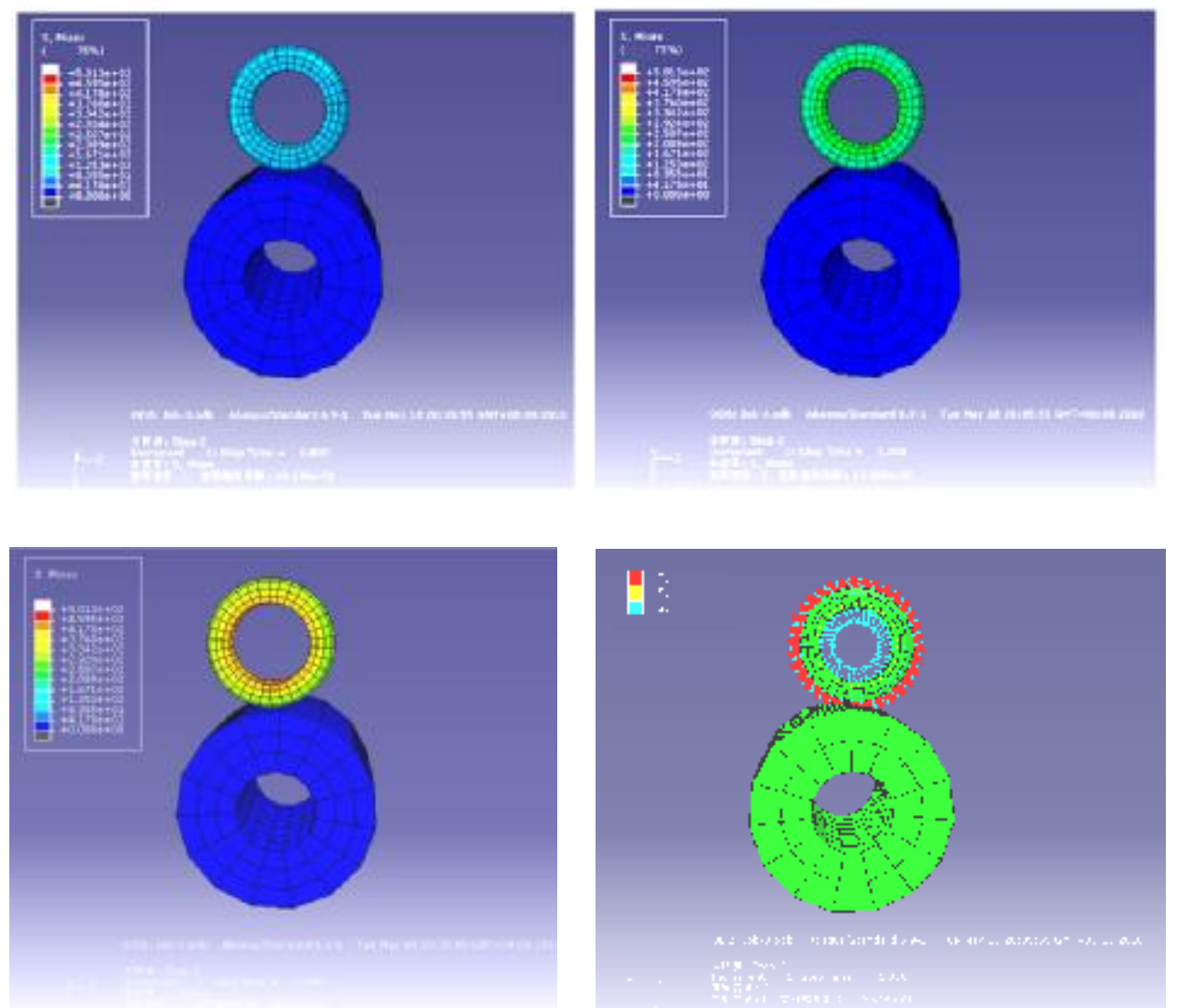

Fig. 2 distortion force

Distortion analysis of reference point. We entered reckoning in result, selected XY data, selected data resource as variable output of ODB field, the variable was selected as distortion, cell junction, plotted datum map, like fig. 3 .
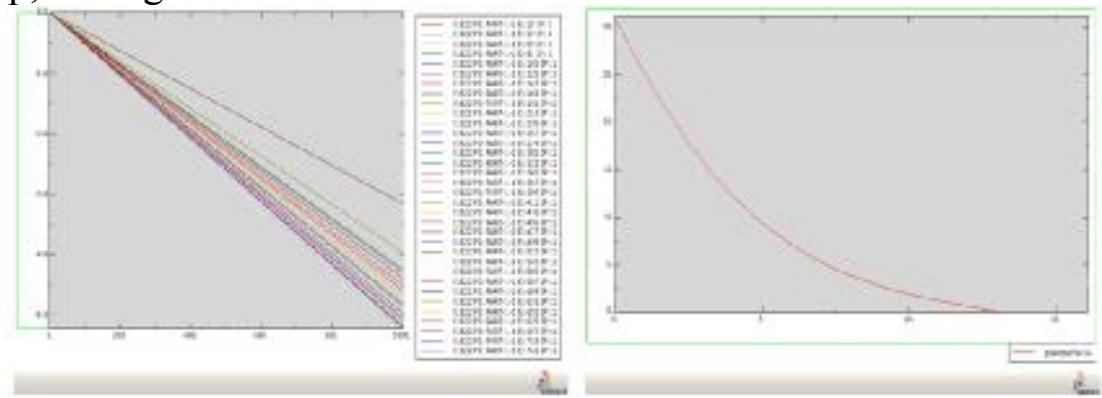

Fig. 3 distortion comeback of the junction 
result analsis on ABAQUS. When comeback time of the tire $T \geq t, t$ represented time of the tire running a circumference, happened standing wave. We specified a angle $\alpha$, distortion resuming completely of the tire in the angle $\alpha$ when speed was $\mathrm{V}$, the effective radius $r$, calculated the critical speed of the tire by distortion of the tire testing, Eq. 1.

$$
V=\frac{\pi r}{T}=\frac{3.14 \times(0.307-0.0103)}{0.01311}=71.06 \mathrm{~m} / \mathrm{s}=255.83 \mathrm{~km} / \mathrm{h}
$$

\section{Standing wave of the tire testing}

We did marker points equably in the circle of the radial tire, used high-speed camera to shoot elasticity distortion of real tire on the automotive chassis dynamometer, shot dynamic pictures with the speed from $150 \mathrm{~km} / \mathrm{h}$ to $180 \mathrm{~km} / \mathrm{h}$, found marker points in the dynamic pictures, utilized AutoCAD to measure marker points, noted the tire radial distortion, angle of the distortion and size of the distortion.

\section{Distortion analysis of the tire rolling}

Size distortion and resume time of the tire. We shot elasticity distortion of the tire by high-speed camera with the speed from $150 \mathrm{~km} / \mathrm{h}$ to $180 \mathrm{~km} / \mathrm{h}$, analyzed radial distortion size and position of elasticity distortion resuming completely, but herein the space restricted, we only researched speed 180 $\mathrm{km} / \mathrm{h}$, this picture was $\pi / 2$ round with continuous distortion, fig 4 . In fig5, black line represented reference round, white line was marker line.

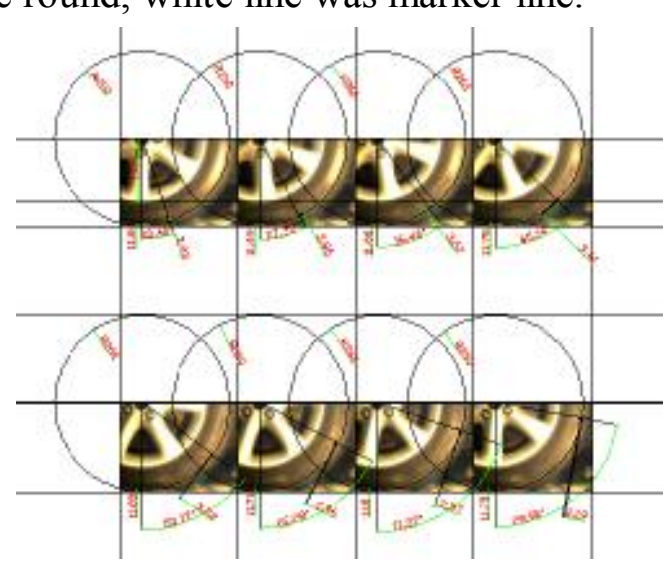

Fig.4 distortion process of marker points

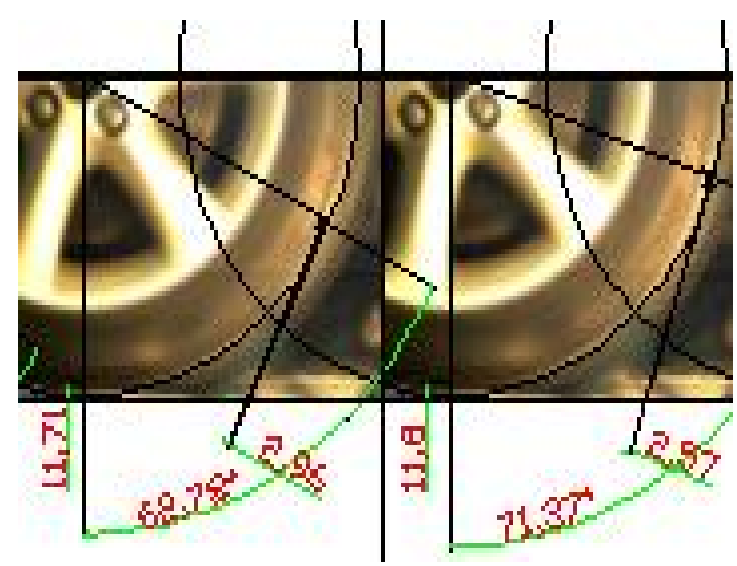

fig.5 megascopic picture

When pressure of the tire was $2.2 \mathrm{MPa}$, load $280 \mathrm{~kg}$, environment temperature $20^{\circ} \mathrm{C}$, size of the radial distortion and time of the elasticity distortion resuming, table1,2.

table 1 size of the radial distortion

\begin{tabular}{|c|c|c|c|c|}
\hline \multirow[t]{2}{*}{ pounts } & \multicolumn{4}{|c|}{ Radial distortion (mm) } \\
\hline & $150 \mathrm{l}: \mathrm{m} / \mathrm{t}$ & $160 \mathrm{~km} / \mathrm{h}$ & 170kwis':a & $\begin{array}{c}-80: \mathrm{ill} \\
\mathrm{h}\end{array}$ \\
\hline 1 & 5.74 & 4. 32 & 3. 95 & 4. 14 \\
\hline 2 & 1. 01 & ล. วล & 3.60 & 3.36 \\
\hline 3 & 3.93 & 3. 62 ? & 3.37 & 3. 76 \\
\hline 4 & 3. 67 & 3. 39 & 3.00 & 3.52 \\
\hline 5 & 3.51 & 3. 02 & 2. 86 & 3. 36 \\
\hline 6 & 3.51 & 2.85 & 2. 69 & 3.06 \\
\hline 7 & 3.37 & 2. $6 \mathrm{ล}$ & 2..55 & 2.69 \\
\hline 8 & 3.10 & 2.38 & 2.23 & 2.36 \\
\hline 9 & $\therefore .97$ & 2. 1ลิ & 1. 97 & \\
\hline 10 & 2.62 & & & \\
\hline
\end{tabular}

bable 2 lime of the elaslicity distorlion resuning

\begin{tabular}{|c|c|c|c|c|}
\hline \multirow[t]{2}{*}{ poirits } & \multicolumn{4}{|c|}{ Time of thdial distmrico resuming $(0.1 \mathrm{~ms})$} \\
\hline & 150 kus $/ \mathrm{h}$ & 160 lin & $170 \mathrm{kta} / \mathrm{h}$ & 180la $/ \mathrm{h}$ \\
\hline $\mathrm{I}$ & 0 & 0 & 0 & 0 \\
\hline 2 & 9. 3 & 9.0 & 9. 3 & s. 7 \\
\hline 3 & 9.3 & 10.0 & 9.7 & 9.0 \\
\hline 1 & 9.3 & 9.0 & 9.0 & 9.3 \\
\hline 5 & 9.7 & 9.3 & 9.0 & 9.7 \\
\hline 6 & 9.0 & 9.7 & 9.7 & 9.3 \\
\hline 7 & 9.3 & 9.0 & 9.0 & 9.0 \\
\hline 8 & 9.3 & 9.3 & 9.3 & 9.3 \\
\hline 9 & 9.3 & 9.7 & 9.7 & \\
\hline 10 & 9.3 & & & \\
\hline
\end{tabular}


Experiment datum disposing. We constructed function of radial distortion and comeback time for different speed, fitting datum of radial distortion and comeback time to calculate time of the radial distortion resuming completely for different speed of the tire. Time of the distortion resuming completely $\mathrm{T} 1=0.0104 \mathrm{~s}, \mathrm{~T} 2=0.0111 \mathrm{~s}, \mathrm{~T} 3=0.0108 \mathrm{~s}, \mathrm{~T} 4=0.0098 \mathrm{~s}$, calculated the time of the tire touching ground for different speed of the tire, $\mathrm{t} 1=0.002049 \mathrm{~s}, \mathrm{t} 2=0.002115 \mathrm{~s}, \mathrm{t} 3=0.001939$, $\mathrm{t} 4=0.001861 \mathrm{~s}$.

So the average time of radial distortion resuming completely of the tire for different speed, Eq. 2:

$$
\begin{aligned}
T & =\frac{\left(T_{1}+t_{1}\right)+T_{2}+t_{2}+T_{3}++t_{3}+T_{4}+t_{4}}{4} \\
& =\frac{0.012449+0.013215+0.012739+0.011661}{4} \\
& =0.012516 \mathrm{~s}
\end{aligned}
$$

Basis on Eq. 1:

$$
V=\frac{\pi r}{T}=\frac{\pi R-H / 3}{T}=\frac{0.2973 \mathrm{~m}}{0.012516 \mathrm{~s}} * 3.6=268.5 \mathrm{~km} / \mathrm{h}
$$

\section{Conclusion}

We constructed simulation model of meridian tire, tested the tire critical speed for standing wave, then constructed test system with high-speed camera, automotive chassis dynamometers and car to test real critical speed, analyzed the pictures of distortion of the tire with high-speed camera shooting by AutoCAD, measured distortion, resuming time and resuming angle for the tire, by fitting datum with size and time of distortion resuming to calculate critical speed of the tire. The method of researching critical speed integrated accuracy of experimentation method and universality of analytical method, held low cost of research, precision of calculating and extensive value of application.

\section{Acknowledgement}

This research was supported by ordinary institutions of higher learning young academic backbone of Heilongjiang Province support program(1252G051).

\section{Reference}

[1] G. Leicester(original), Zhang Yinghong(translation) .Research and development of automobile tire[M]. beijing: China Machine Press, 2012.

[2] Chang Y P , Gindy M E , Streit D A. Influence of tyre loading and inflation pressure on standing waves phenomenon using PAM-SHOCK[J]. Heavy Vehicle Systems, 2003, 10(1/2):862111.

[3] Anindya C , Joseph P C , John D. On contact-induced standing waves in rotating tires: experiment and theory[J]. Journal of Sound and Vibration, 1999, 227 (5):104921081.

[4] Wang Qiang, Zhang Na, Shi Yuhuan, Qi Xiaojie, Wang Donghao. Study of standing wave critical speed of radial tire based on ABAQUS[J]. Journal of Jiamusi University ( Natural Science Edition), Vol.31, No.5, PP.722-725, 2013.

[5] Wang Yunlong, Qi Xiaojie, Lv Degang, Wang Guotian. Simulation of modeling and standing wave for the radial tire. Journal of Heilongjiang Institute of Technology, Vol.26, No.3, PP. 14-17, 2012.

[6] J. R. Choa, * , K. W. Kimb, H. S. Jeongb. Numerical investigation of tire standing wave using 3-D patterned tire model[J].ScienceDirect, 2007, (6).

[7] Wang Qiang, Qi Xiaojie, Wang Yunlong, Lv Degang, Wang Guotian. Finite element analysis on critical speed of standing wave for high-speed tire. Vehicle \& Power Technology, No.4, PP. 46-50, 2011. 
